\section{Studien zum Weber-Paradigma}

Herausgegeben von

Gert Albert

Agathe Bienfait

Steffen Sigmund

Mateusz Stachura

Mit der Reihe "Studien zum Weber-Paradigma" soll ein ort für solche Publikationen geschaffen werden, die sich in Interpretationen, theoretischen weiterentwicklungen und empirischen Studien mit dem Werk Max Webers auseinandersetzen. Die Bezugnahme auf das Webersche Forschungsprogramm schließt dessen kritische Diskussion durch Vertreter anderer theoretischer Positionen mit ein: Institutionentheoretische Fontführungen, ethische und sozialontologische Fragen im Gefolge Weberscher Unterscheidungen wie auch neue oder alte verbindungen Weberianischer Theorie mit philosophischen Strömungen werden diskutiert. Die "Studien zum weber-Paradigma" sind einem undogmatischen und innovativen Umgang mit dem Weberschen Erbe verpflichtet.
Gert Albert · Agathe Bienfait Steffen Sigmund Mateusz Stachura (Hrsg.)

\section{Aspekte des Weber-Paradigmas}

Festschrift für Wolfgang Schluchter

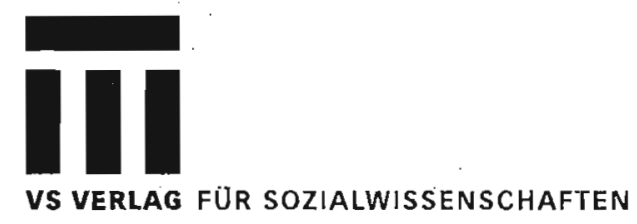




\title{
Weber, Sombart und die neuere Kapitalismustheorie
}

\author{
Markus Pohlmann
}

Die Kapitalismusliteratur der letzten drei Jahrzehnte ist von einer eigenartigen Kluft gekennzeichnet. Während sich im Schnittpunkt von Geschichte und Soziologie immer mehr makrostrukturelle Ansätze formiert haben, welche die „lange Dauer" des Kapitalismus in den Blick nehmen, erweist sich die gegenwärtige kapitalismustheoretische Trendliteratur davon weitgehend abgekoppelt. Nur ganz wenige Autoren bilden heute Brücken zwischen diesen Kristallisationspunkten der kapitalismustheoretischen Diskussion. War die soziologische Kapitalismustheorie in Deutschland immer auch sozio-historisch verankert und ihre Gegenwartsanálysen mit sozio-historischen Erklärungen versehen, so beginnt sich in jüngster Zeit diese Verankerung zu lockern. Dies geschieht paradoxerweise zu einem Zeitpunkt, zu dem sich neue sozio-historische Ansätze mit empirisch vertieften Erklärungen des historischen Phänomens Kapitalismus hervorwagen. Die dadurch entstandene Kluft ist um so erstaunlicher, als in beiden Diskussionsschwerpunkten nahezu gleichlautende Korrekturen an zentralen Prämissen der älteren Kapitalismustheorie, also an Marx', Webers und Sombarts Ansätzen angebracht werden. Insbesondere wird in beiden Diskussionsschwerpunkten die historische Überholtheit vieler Annahmen der deutschen Kapitalismustheorie vorgeführt. Diese Beriuhrungspunkte wurden aber bislang von beiden Seiten weder beachfet noch von dritter Seite herausgearbeitet ${ }^{1}$. Die sozio-historischen Arbeiten nehmen trotz vielfacher Anstrengungen, ihre Ergebnisse zu aktualisieren, kaum Bezug auf die gegenwärtige Kapitalismusliteratur, und diese fundiert ihre Diagnosen und Trendannahmen so gut wie nie mit weiter zurückreichenden historischen Befunden.

Ich möchte im folgenden zeigen, dass Webers und Sombarts Ansätze bei aller historischen Gebundenheit ihrer Annahmen eine Brücke bilden können, um die sozio-historische Analyse des Kapitalismus mit seinen Gegenwartsdiagnosen zu verbinden. Insbesondere Max Webers Theorie bietet für eine solche wechselseitige Bezugnahme ein tragfähiges Fundament. Mit ihrer Fortführung kann einer solchen Verknüpfung ein differenzierungstheoretisches Verständnis kapita-

\footnotetext{
${ }^{1}$ Diese Defizite sind auch deswegen erstaunlich, weil die Vertreter beider Ansätze häufig in demselben Sprach- und Kulturraum angesiedelt sind. Mit einigen wenigen Ausnahmen, wie z.B. der französischen Annalenschule, sind die meisten Autoren der sozio-historischen Kapitalismustheorie im anglo-amerikanischen Raum verankert (vgl. Vester 1995: 2). Dasselbe gilt auch für die gegenwartsdiagnostische Trendliteratur zum Thema Kapitalismus.
} 
listischer Entwicklung zugrundegelegt werden, das sich von vielen „Gleichungen" und "Gesetzen" des Mainstreams der älteren Kapitalismustheorie verabschiedet. Auf diese Weise lässt sich die Kapitalismustheorie als Wirtschaftssoziologie reformulieren. Eine solche Reformulierung soll aus einigen konzeptionellen Sackgassen der älteren Kapitalismustheorie heraushelfen und es erlauben, von ihrer gesellschaftstheoretischen Überfrachtung Abstand zu nehmen, ohne auf gesellschaftstheoretische Bezüge verzichten zu müssen. Sie muss sich dazu allerdings über das wechselseitige Dementi von Kapitalismustheorie und Differenzierungstheorie ${ }^{2}$ hinwegsetzen. Inwiefern dies unter Rückbezug auf Weber und Sombart gelingen kann, ist Gegenstand der folgenden Ausführungen. Sie sind als Vorarbeiten für eine Fundierung, theoretische Rahmung und Verankerung der Kapitalismustheorie in der modernen Wirtschaftssoziologie gedacht.

Ich werde zunächst den Ausgangspunkt meiner Überlegungen schildern, nämlich die deutsche Kapitalismustheorie zu ihrer Blütezeit im ersten Viertel des vergangenen Jahrhunderts (1) und kurz vorstellen, was die Weberschen und Sombartschen Perspektiven vom marxorientierten Mainstream unterscheidet und wo sie mit diesem konform gehen. Im Anschluss daran werden die Besonderhei ten ihrer beiden Ansätze vor dem Hintergrund diskutiert, inwiefern sie der neueren Kapitalismusliteratur eine theoretische Perspektive geben und helfen können, ihre verschiedenen Befunde in dieser Perspektive stärker zu verzahnen (2). Dies geschieht unter Bezugnahme auf ein Verständnis des Kapitalismus als eine koexistierende Wirtschaftsweise (2.1), auf die Möglichkeiten der Verknüpfung von Handlungs und Strukturebene (2.2), auf die Theorie des okzidentalen Rationalismus als konzeptionelle Klammer (2.3) und auf Entwicklungsvorstellungen, die differenzierungstheoretisch, inspiriert die Zukunft offen halten (2.4), um abschließend zu resümieren, wo ein differenzierungstheoretisches Verständnis des Kapitalismus seine Ansatzpunkte findet (3).

${ }^{2}$ Für Niklas Luhmann war der Begriff des Kapitalismus ein sozialgeschichtlicher, der wirtschaftswissenschaftliche Details ungeklärt lasse und eine weithin veraltete Beschreibung einer Gesellschaft mit ausdifferenziertem Wirtschaftssystem anbiete. „Sie enthält Nebenbedeutungen und mobilisiert Vorwurfshattungen", so Luhmann, die darauf abzielen, das politische System der Gesellschaft zu beein(192) Die Prob : 192 Preble Kapitalismus jedenfalls „ganz unzureichend "formuliert (ebd.. 198). Wallerstein hingegen hält die Behauptung, dass ,soziale Realität in [...] unterschiedlichen und getrennten Bereichen", z.B. im politischen, wirtschaftlichen und sozio-kulturellen, vorkommt, für eine "schreckliche Erbschaft" der Sozialwiśsenschaften des 19. Jahrhunderts (Wallerstein 1995: 313). Denn dieses Begreifen von Differenzierung verkenne, dass das "Ganze" ein ,nahtloses Geflecht" sei und habe heute keinen. "heuristischen intellektuellen Wert" mehr (ebd.: 314). Er plädiert deshalb für eine holistische Analyse (vgl. ebd.: $314 \mathrm{f}$ )

\section{Der Ausgangspunkt: Die Blütezeit der Kapitalismustheorie in} Deutschland

1873 schreibt Karl Marx im Nachwort zur zweiten Auflage des Kapitals: „Die politische Ökonomie blieb in Deutschland bis zu dieser Stunde eine ausländische Wissenschaft. (...) Wie zur klassischen Zeit der bürgerlichen Ökonomie blieben die Deutschen auch zur Zeit ihres Verfalls bloße Schüler, Nachbeter und ,Nachtreter', Kleinhausierer des ausländischen Großgeschäfts" (Marx 1873; 19-21). Dies änderte sich mit und nach Marx. Mit ihm als Vor]äufer (und Schumpeter als Nachzügler) ist die Kapitalismustheorie auch heute noch von Perspektiven geprägt, die hauptsächlich dem ersten Viertel des vergangenen Jahrhunderts entstammen. Diese Phase lässt sich (mit Ausnahme des Vorläufers Marx und des Nachzüglers Schumpeter) mit dem Erscheinen der ersten (1902) und der letzten Fassung (1927) von Sombarts "Der moderne Kapitalismus" zeitlich einordnen (siehe Anhang I). Im Strom der seit dem 19. Jahrhundert immer stärker anschwellenden Kapitalismusliteratur finden sich hier einige - wenn auch nicht alle - der entscheidenden Klippen der sozialwissenschaftlichen Kapitalismusinterpretation. Es ist, so möchte ich diese Phase in, leicht ironischem Anklang an Hegels Vorlesungen über die Philosophie der Weltgeschichte nennen, die "Blütezeit der deutschen Kapitalismustheorie". Alle hier behandelten, klassisch gewordenen Autoren dieser Zeit sind im deutschsprachigen Raum angesiedelt oder in ihrem Werk den Diskussionen in diesem Raum verpflichtet.

Diese „soziologische Klassik" der Kapitalismustheorie hatte mit Marx und Weber einen doppelten paradigmatischen Kern mit ganz unterschiedlichen Anschlussfähigkeiten für ein differenzierungstheoretisches Verständnis des Kapitalismus ${ }^{4}$. Marx und seine Nachfolger wählten mit dem von Hegel inspirierten Produktionsbegriff ein Theoriedesign, das quer zur Differenzierungstheorie angelegt war - obwohl Marx' historische Theorie Differenzierungsprozesse sehr präzise im Blick hatte (vgl. DI, MEW 3: 60 f.; MkP, MEW 4: 463 ff.). Marx grenzte den Kapitalismus als historisch neue Epoche von vorbürgerlichen, vor-

${ }^{3}$ Mit dem Begriff der ,soziologischen Klassik" der Kapitalismustheorie sind in wesentlichen zwei Abgrenzungen verbunden. Anvisiert sind zum einen Kapitalismustheorien, deren primäres Erklärungsziel nicht auf Wirkungsgesetze der Ökonomie, sondern auf deren Wirkweisen für die Konstiturungsziel nicht auf Wirkungsgesetze der Okonomie, sondern auf deren Wirkweisen für die Konstitu-
tion unid den Wandel des Sozialen, insbesondere der Gesellschaft, gerichtet ist (vgl. Parsons 1928: tion unid den Wandel des Sozialen, insbesondere der Gesellschaft, gerichtet ist (vgl. Parsons 1928:
641 ). Zum anderen sollen diese Ansätze darüber hinaus - dies spricht der Begriff der "Klassik" an 641). Zum anderen sollen diese Ansätze darüber hinaus - dies spricht der Begriff der "Klassik" an -
zum nur schwer hintergehbaren Kanon der Diskussion des Fachs gehören, d.h. ein Paradigma oder zum nur schwer hintergehbaren Kanon der Diskussion des Fachs gehören, d.h. ein Paradigma
mehrere abbilden, welche die Standards der soziologischen Diskussion weitgehend geprägt haben.

${ }^{4}$ Es handelt sich hier um die Darlegung eines eher grundlegenden Verständnisses von Kapitalismus, weswegen ich den Singular verwende, ohne damit abzuerkennen, dass es empirisch-konkret oft sinnvoller ist, im Plural, also von Kapitalismen, zu sprechen. 
kapitalistischen Epochen ab und rief ihn als einen Weltkapitalismus aus, in dem die entwickelteren Staaten den unterentwickelten das Bild ihrer eigenen Zukunft zeigten. Webers Nähe zu differenzierungstheoretischen Annahmen ließ ihn im Kapitalismus hingegen nur ein Ordnungsprinzip der Wirtschaft sehen, dass in unterschiedlichen Kulturen und zu unterschiedlichen Zeiten zur Geltung kam, aber in der europäischen Moderne seine systemische Ausprägung erfuhr. Auch für das weniger systematische Theorieangebot von Sombart gilt, dass seine Anschlussfähigkeit für ein differenzierungstheoretisches Verständnis des Kapitalismus hoch ist. Er räumt nicht nur mit der Gleichsetzung von Kapitalismus und Gesellschaft, sondern auch mit jener von Kapitalismus und Wirtschaft auf - auch wenn sein Werk in vielen anderen Aspekten die marxorientierten Ideen der soziologischen Klassik der Kapitalismustheorie teilt. Trotz aller Differenzen gab es aber auch eine gemeinsame Interpretationslinie, welche die Ausdifferenzierung des Systems des 'modernen' Kapitalismus seit dem 16. Jahrhundert anheben sieht und seine hohe, wenn nicht höchste Entwicklung historisch-konkret mit Industrialisierung, Massenproduktion und Großunternehmen verbindet (KI: 161; vgl. dazu auch Pohlmann 1996)

Marx' Ziel, die Bewegungsgesetze der bürgerlichen Gesellschaft aufzudecken (KI: 15 f.), führte im Mainstream der deutschen Kapitalismustheorie dazu, eine analytische Unterscheidung zwischen Wirtschaftsform und Gesellschaftsform in der Regel nicht vorzunehmen. Die Wirtschaftsform der kapitalistischen Produktionsweise wurde mit der gesamten Gesellschaftsformation gleichgesetzt Marx' geschichtsphilosophisch inspiriertes, anthropologisches und gesellschaftsbezogenes Begreifen des „Produktionsprozesses“, in dem Gesellschaft und Ökonomie als Unterschiedenes aufgehoben waren, erweis sich als Schule machend. Die gewählte Losung 5 war einfach: Die Entwicklung von Wirtschaft und Gesellschaft lässt sich nur durch den epochemachenden Kapitalismus und seine Bewegungsgesetze begreifen.

Auch die Orientierung an Marx' Vorgabe einer Herrschaft des Industriekapitals ist in dieser Tradition sehr stark. Allerdings wird Marx' Leitthema - mit der Ausnahme von Rosa Luxemburg - konsequent in eine Theorie des kartellierten, organisierten Finanzkapitalismus überführt. Kapitalismus in seinem Höchststadium heißt in dieser Tradition der deutschen Kapitalismustheorie, dass unterschiedliche Wirtschaftsweisen und Industrieformen sich unterordnen oder untergehen - das Handelskapital, der Agrarsektor, die Klein- und Mittelunternehmen, das Handwerk etc. Der „Konkurrenzkapitalismus“ wird auf allen Ebenen der

${ }^{s}$ Mit der Ausnahine von Rosa Luxemburg, die auf Basis der Auseinandersetzung mil dem Marxschen Modell der erweiterten Reproduktion zu einer konkret-historischen Identifikation der kapitalisschen Modell der erweiterten Reproduktion zu einer konkret-historischen Identifikation der kapitalis-
tischen Produktionsweise und damit zu dem Schluss kommt, dass diese noch keineswegs die wirtschaftich vorherrschende sei (vgl. Luxemburg 1913).
Wirtschafts- und Geselischaftsorganisation durch den organisierten Kapitalismus, durch Oligopole und Monopole ersetzt. Hier greift eindrucksvoll, was Sombart zurecht an der Marxschen Theorie als ,,Sozial-Monismus" beklagte: Ein Wirtschaftssystem, eine Produktionsweise wird durch eine andere abgelöst. Ein Pluralismus verschiedener Formen ist aufgrund des Hegelschen Erbes theoriearchitektonisch nicht denkbar. Und so ergibt sich aus Gleichungen und Negationen jene eigentümliche, wohl bekannte Theoriemechanik, nach der sich eine Produktionsweise mit einer Form des Klassengegensatzes so durchsetzt, dass alle anderen Produktionsweisen, Sektoren, Klassenlagen untergehen, um in dieser Einförmigkeit als höchst entwickelter Kapitalismus schließlich selbst negiert werden zu können. Auf jeder Ebene, in jeder Dimension und schließlich auch in jeder Region der Welt wird diese Gesellschaft als kapitalistische identifizierbar, mit diesem gleichgesetzt - bis zur endlich erreichten Transformation in einen wie auch immer gearteten Sozialismus.

Diese Höchstform des Kapitalismus fand, dann auch für Weber, Sombart und Schumpeter, ihren untrüglichen Ausdruck in der großindustriell organisierten Massenproduktion. Bei Marx und in der Nachfolge Marxens, z.B. bei Hilferding (1910), Luxemburg (1913) und Lenin (1917), steht die industrielle (bzw. finanzkapitalistische) Organisationsform der Kapitalakkumulation mit der Notwendigkeit expansiver - sozial und territorial organisierter - Unterdrückung und Ausbeutung der Arbeiterklasse, der entstandenen proletarischen Schichten im Vordergrund ${ }^{6}$. Im Gegensatz dazu rücken Sombart (1927) und Weber (1920) und im Anschluss daran Schumpeter (1942) - die Konsequenzen der bürokratischen großindustriellen Organisation für die Unternehmerklasse und die bürgerlichen Schichten in den Mittelpunkt. Die gemeinsame Diagnose orientiert sich bei den drei letztgenannten Autoren aber eher (mit ganz unterschiedlicher Betonung) am möglichen Zugrundegehen einer ,unternehmerischen“ Wirtschiaftsgesinnung und sinkenden Chancen, familiale sowie andere materiale Orientierungen gegenüber der bürokratischen, formal rationalen Organisation zu behaupten.

Aber zugleich galt: Nie waren sich die Kapitalismustheoretiker über das Ende des Kapitalismus sicherer, als während der Heraufkunft der großindustriellen Massenproduktion. Im Zentrum des Begreifens von kapitalistischer Entwick-

${ }^{6}$ Die große Industrie letztlich ist es für Marx, die mit „hartem Stoß" die endgültige Befreiung von den feudalen Fesseln bewirkt. Ihre Produktivkraft sprengte die Produktionsverhältnisse. Für Marx und Engels vollendete sie eine Entwicklung, die Vernichtung und Zerstörung (von naturwüchsigen Verhältnissen, aller übrigen Klassen, aber auch von Ideologie, Moral und Religion), Auflösung (in Geld- und Kapitalverhältnisse), Zwang und Subsumtion (unter das Kapital und die universelle Konbedeutete (vgl. DI, MEW 3: 60 f.; vgl. dazu auch: MkP, MEW 4: 463 ff.). Erst die große Industrie bringt die soziale Revolution der kapitalistischen Produktion auf ihren dramatischen Höhe- und Endpunkt. 
lung steht eine sozialgeschichtliche Fassung, die sich auf Entstehung und Untergang konzentriert. So halten fast alle Autoren dieser Phase (am wenigsten aber Max Weber) den modernen Kapitalismus in dieser Ausprägung für transitorisch - für ein System, das untergehen muss, d.h. sich mit mehr oder weniger großer Wahrscheinlichkeit in einen Sozialismus oder eine Diktatur der Beamten verwandeln wird. Dafür zeichnete für die Klassik der Kapitalismustheorie typischerweise der Erfolg kapitalistischer Entwicklung verantwortlich, nicht ihr Scheitern.

Was nun aber waren die Besonderheiten der Sombartschen und Weberschen Ansätze und inwiefern sind sie geeignet, den Revisionen der angloamerikanischen Kapitalismusiteratur einen verbindenden theoretischen Rahmen zu geben?

\section{Sombart, Weber und das differenzierungstheoretische Verständnis des} Kapitalismus

Sombart erschien der Hochkapitalismus als eine ,absonderliche Episode in der Geschichte der Menschheit, die diese vielleicht nur geträumt" habe (MK III,1: XIV). Sein Werk ist für uns vor allem interessant, weil es bei aller Zwiespältigkeit mit wichtigen Vorstellungen der marxorientierten Linie in der deutschen Kapitalismustheorie in einer Art bricht, die für ein modernes wirtschaftssoziologisches Verständnis des Kapitalismus anschlussfähig ist.

\subsection{Der Kapitalismus: Von der Alleinherrschaft zur Koexistenz verschiedener} Wirtschaftsweisen

In theoretischer Perspektive betrachtet Sombart den Kapitalismus als eine Verkehrsform unter anderen, die sich von Handwerk und Eigenwirtschaft durch das Erwerbsprinzip (im Gegensatz zur Bedarfsdeckung) und den ökonomischen Rationalismus (im Gegensatz zum ökonomischen Traditionalismus) als Wirtschaftsgesinnung unterscheidet. Diese einfache Unterscheidung, die. seinem Werk zugrunde liegt, hat weitreichende Konsequenzen. Die Vorstellung einer Vorherrschaft des Kapitalismus wird damit von einer unhinterfragten Prämisse des Denkens zu einer empirisch zu überprüfenden Annahme. Damit verliert sie den Charakter ihrer bloßen theoretischen Deduktion und begründet eine Stoppregel gegenüber ungeprüften Gleichsetzungen von Kapitalismus, Wirtschaft und Gesellschaft Auch daran anschließende Perspektiven eines selbstverständlich vorausgesetzten Primats der Ökonomie, begründet durch die gesellschaftliche
Herrschaft des Kapitalismus oder des Kapitalismus als treibende Kraft gesellschaftlicher Entwicklung bedürfen nun eines empirischen Nachweises. Sombart, der viel Aufwand betrieb, um eine konkret-historische Fundierung der unterschiedlichen Wirtschaftsweisen in ihrem Zusammenspiel zu leisten, kommt dagegen am Ende zu dem Schluss: „All diejenigen irren, die für die Zukunft die Alleinherrschaft eines Wirtschaftssystems voraussagen" (MK III,2: 1008). Im Verlauf der Geschichte gestalte sich das Wirtschaftsleben vielmehr immer reicher, neue Stimmen träten hinzu, ohne dass alte aufhörten zu klingen. Mit diesem Befund nimmt Sombart Abschied von der Marxschen Theorie dialektischer kapitalistischer Entwicklung. Unterschiedliche Wirtschaftsweisen wie z.B. Handwerk, Eigenwirtschaft, Genossenschaftswirtschaft, Kapitalismus bestehen nach Sombart nebeneinander fort. Die Deduktion ihres Untergang bei der Fortentwicklung des Kapitalismus, zentrales Theorieprinzip bei Marx, wird bei Sornbart als 'Annahme aufgegeben, da sie nur noch kontrafaktisch aufrechterhalten werden konnte. Wie immer plausibel seine empirische Begründung ${ }^{7}$ erscheinen mag - und die Wirtschaftsgeschichte sowie die Wirtschaftssoziologie geben ihm heute recht - sie führt Sombart dazu, sehr sorgfältig zwischen Gesellschaft, Wirtschaftsystemen und Kapitalismus als einer Wirtschaftsweise unter anderen zu unterscheiden. Damit bietet er nicht nur einen Grundstein für ein modernes wirtschaftssoziologisches Begreifen des Kapitalismus, sondern auch ein Verständnis desselben, das die Revisionen der sozio-historischen und gegenwartsdiagnostischen Kapitalismusliteratur zu instruieren vermag.

Braudel hat in seinen Arbeiten zur Theorie kapitalistischer Entwicklung viel Kritik an den Traditionen der deutschen Kapitalismustheorie geäußert und insbesondere seine Distanzen zu den Vorstellungen von Marx, Weber und Sombart herausgestrichen. Seine Theorie ${ }^{8}$ lässt sich dennoch am ehesten in der Tradition der Weberschen und Sombartschen Linie der Interpretation als Theorie der Systemdifferenzierung rekonstruieren. So ist Kapitalismus in Braudels historischem Ansetzen ebenfalls nur eine partielle Form des Ökonomischen, ein Stratum in einer intern differenzierten Wirtschaft, die ihren Schwerpunkt im alltäglichen

${ }^{7}$ Für die Epoche des Hochkapitalismus weist Sombart anhand der Berufs- und Gewerbestatistik darauf hin, dass, in ganz groben Umrissen der Anteil des Kapitalismus. an dem Wirtschaftsleben West- und Mittelen gas a

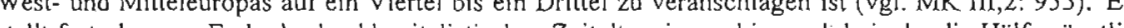
sellt fest, dass am Ende des hochkapltalistischen Zeitalers inmerin noch beinahe die Halfe samtli cher Erwerbstätiǵen - ohne die Landwirtschaft - handwerksmäßig beschäftigt ist (vgl. ebd.: 963). Er merkt jedoch an, dass das Herrschaftsgebiet des Kapitalismus - gemessen an seinern Anteil am gesellschaflichen Gesamterzeugnis - größer ist als es die Anzahl der "ihm verfallenen Personen" erkennen lässt (vgl. MK III,2: 956).

Bei der Herausarbeitung seiner Erklärungsprinzipien kapitalistischer Entwicklung gerät die In terpretation zwangsläufig zur rationalen Rekonstruktion, da Braudel eher ein Anlıänger cler Kunst der Beschreibung, als von "großer Theorie" war. 
materiellen Leben hat ${ }^{9}$. Für Braudel, der von der Pluralität der Gesellschaft ausgeht, erscheint es genauso unsinnig, die gesamte Gesellschaft Europas zwischen dem 11. und 15. Jahrhundert als „Feudalismus" zu fassen, wie in den folgenden Jahrhunderten als "Kapitalismus" (vgl. Braudel 1979b/90: 512). Jedes Mal wird ein Stratum der Gesellschaft oder eine soziale Hierarchie in unzulässiger Weise zur Charakterisierung des "Ganzen" benutzt. Jedes Mal wird Einzahl verwendet, wo Plural und Koexistenz von Ordnungen, Systemen und Produktionsweisen angebracht wäre (vgl. ebd.: 513). Auch wenn er daran andere Unterscheidungen als Sombart anschließt, ist es doch diese Rücknahme gegenüber einem überfrachteten, einer spezifischen historischen Phase gedankten Kapitalismusbegriff, die es ermöglicht, die „lange Dauer" des Kapitalismus in den Blick zu nehmen. Deswegen konzediert auch Michael Mann in seinen historischen Analysen, dass ältere Epochen jeweils Nischen für deri Kapitalismus bereit hielten, auch wenn es eine Gesellschaft, in der diese Aktivitäten dominiert hätten, vor der Moderne nicht gegeben habe (vgl. Mann 1991: 204). Gleichwohl verabschiedet auch Mann in seinen historischen Analysen die Vorstellung eines irgendwie gearteten gesellschaftlichen Primats ökonomischer Organisationsformen. Je ,institutioneller" die Geschichte des Sozialen wird, desto unwahrscheinlicher wird in Manns Augen auch empirisch der Primat oder gar die Exklusivität einer Organisationsform (vgl. Mann 1991: 401 ff.).

Neben dieser historisch differenzierteren Eingrenzung des Kapitalismus wird in der sozio-historischen Diskussion auch eine Abkehr von einem totalisierenden und/oder nationalstaatlich verengten Begriff von Gesellschaft erkennbar. Dieser erscheint vielen Autoren als irreführend, weil er Grenzen voraussetzt, wo keine sind und so einen historischen Vergleich auf Basis falscher Abstraktionen anregt. Dabei spielt vor allem eine Rolle, dass es prinzipiell mehrere Formen von Vergesellschaftung - und eben nicht nur jene durch Politik und Recht konstitutierte - gibt und eine analytische Verengung auf diese ein Missverstehen historischer Entwicklungen geradezu provoziert. Die instruktive Abkehr von der Gleichsetzung von Nationalstaat und Gesellschaft bedeutet jedoch nicht, dass Nationalstaaten keine Rolle mehr spielten. Vielmehr macht diese Trennung erst möglich zu bestimmen, inwieweit sie das Prozessieren einer differenzierten Gesellschaft konditionieren (vgl. Skocpol 1979; Runciman 1989: 5, Vester 1995: 111; Crow 1997: $14 \mathrm{f}$.).

In diesem differenzierten Kapitalismusverständnis trifft sich die soziohistorische mit der gegenwartsbezogenen Kapitalismusliteratur. Für diese ist empirisch ohnehin klar, dass sich wirtschaftliche und geselischaftliche Entwick-

${ }^{9}$ Braudel versteht den Kapitalismus weder per se als Verkörperung der Gesellschaft noch der Wirtschaft der Gesellschaft. Weder durchdringt er die ganze Wirtschaft noch ist er notwendigerweise an eine bestimmte Gesellschaftsformation geknüpft oder bestimmt diese (vgl. Braudel 1986: 60). lung zunehmend entkoppeln und der Kapitalismus andere Wirtschaftsformen nicht zum Verschwinden gebracht hat, sondern mit ihnen koexistiert (vgl. Reich 1993; Strange 1997). Die theoretische Gleichsetzung von Kapitalismus und Gesellschaft ist deswegen zögerlicher geworden. Darin weist die neuere angloamerikanische Kapitalismusliteratur eher auf Weber und Sombart zurück, als sich noch mit dem Produktionsbegriff auf die Erklärung der Anatomie der bürgerlichen Gesellschaft zu versteifen. Ihre Beobachtungen werden jedoch nicht in ein systematisches differenzierungstheoretisches Verständnis des Kapitalismus überführt, das man im Rückgriff auf deren Ansätze gewinnen kann. Eine Perspektive auf den Kapitalismus als eine Verkehrsform unter anderen, gepaart mit einer Rücknahme der Gleichsetzung von Kapitalismus und Gesellschaft sowie einer Skepsis gegenüber einem falsch verstandenen Gesellschaftsbegriff, wie sie auch Webers Analysen kennzeichnete, eröffnet eine andere Perspektive der Kapitalismusinterpretation. Man muss dann die historische Kontingenz bestimmter Formen des Kapitalismus angemessen berücksichtigen und kann nicht mehr einfach die Wirtschaft oder die Gesellschaft mit einer bestimmten Ausprägung des Kapitalismus historisch gleichsetzen. Dadurch wird die historische Varianz des Kapitalismus sichtbar und interpretierbar, die Frage seiner Dominanz empirisch stellbar. Der Bezugspunkt der Interpretation wechselt dann von einer totalisierenden Folgenabschätzung (Ausbeutung, Entfremdung, Rassismus, Sexismus etc.) auf den "Kampf" um seine Geltungsbereiche. Denn diese müssen sowohl innerhalb der Wirtschaft als auch innerhalb der Gesellschaft historisch erschlossen und institutionell behauptet werden. Genau darauf kann nun eine differenzierungstheoretische Kapitalismustheorie ihr Augenmerk richten und die historischen wie die aktuellen Befunde dazu neu sortieren und zusammenbinden. Der Kapitalismus erscheint in dieser Perspektive als ein „,contested terrain " gesellschaftlicher Entwicklung, auf das die Interpretation seiner Wirkungen bezogen werden muss. Erst dann kann man diskutieren, unter welchen historischkonkreten institutionellen Bedingungen sich eine "Ökonomisierung" von anderen gesellschaftlichen Wertsphären durchsetzt und unter welchen eine solche „Kolonialisierung" durch den Kapitalismus nicht gelingt. Die Konsequenz der Übernahme einer solchen Vorstellung ist also, dass man sich um die Bedingungen und Mittel dieses Kampfes um die Geitungsbereiche des Kapitalismus kümmern muss und um die institutionellen Konfigurationen, welche die Verwirklichung von kapitalistischen Geltungsansprüchen ermöglichen. In dieser Perspektive kann die Kapitalismustheorie einen wichtigen Beitrag zur modernen Wirtschaftssoziologie leisten, auch wenn sie hier erst am Anfang steht. 


\subsection{Die Ausdifferenzierung der modernen Wirtschaft und die Verknüpfung von Handlungs- und Strukturebene}

Sombart macht darüber hinaus, ebenso wie Weber, in differenzierungstheoretischer Weise klar, was er u.a. als spezifische Qualität des Hochkapitalismus sieht: dass dieser der Gängelung durch die Staatsorgane entwachsen ist ${ }^{10}$. Weber entwickelt auf dieser Basis ein differentexungstheoretisches Verständnis des Kapitalismus, das ein konzises Angebot zur Verknüpfung der Handlungs- und Struk turebene in der historischen Entwicklung macht (vgl, zur Einordnung auch Schluchter 2000: $125 \mathrm{ff}$. und für eine wissenschaftstheoretische Grundlegung Albert 2005) und sich darin sowohl vom Mainstream der älteren Kapitalismustheorie als auch von der neueren Differenzierungstheorie unterscheidet. In dieser Verknüpfung von Handlungs- und Strukturebene, deren genauer theoriearchitektonischer Aufbau in Webers „Soziologischen Grundbegriffen“ dargelegt wird, ist ein zweiter wichtiger Baustein für ein modernes wirtschaftssoziologisches Verständnis des Kapitalismus zu sehen. Der Kapitalismus im Altertum, in der Polis, lebte nach Weber letztlich doch allein vom Politischen und war nur ,indirekt ökonomisch" (SWG: 263 ff.). Er hing an der privaten Ausbeutung politischer Herrschaftsverhältnisse in einem expansiven Stadtstaat und stockte mit dem Wegfall dieser Quelle (vgl. ebd.: 276). Damit ein Differenzierungsprozess in Form der Trennung von Familie und Gewerbe und der Schaffung von zusammengefassten künstlichen Betriebseinheiten stattfinden konnte, musste also wiederum in historischer Kontingenz der stabile Absatzmarkt die Entwicklung eines Kalküls ermöglichen, in dem sich ein spezialisiertes Produktions- und Kapitalverwertungsinteresse mit einem Ordnungsprinzip der Produktion (spezialisierte Produktion und Organisation freier Arbeit) verband. Dieser Absatzmarkt diente historisch als Ersatz für die private Ausbeutung politischer Herrschaftsverhältnisse, was wiederum die Freisetzung der Wirtschaft von politischer Intervention und Annektion, ihre Depolitisierung im nächsten Schritt möglich machte. Der Schutz des reinen ökonomischen Kalkuils vor direkter politischer Einflussnahme war dadurch in der historischen Tendenz soziologisch wahrscheinlicher geworden.

\footnotetext{
${ }^{10}$ Die Charakteristika der funktionalen Ausdifferenzierung des Wirtschaftssystems, insbesondere seiner De-Politisierung, fundierten auch Webers begriffliche Fassung des modernen rationalen Kapitalisurs als "bïgerlich " (ven Kapitalismus als, „buigenticher Betriebskapitalismus" mil ", RSI: 10). Gegenüber dem jüdischen Paria-Kapitalismus, dem Abenteurer-, Beute- und politischen Kapitalismus, dem wucherischen oder spekulativem Kapitalismus, für Weber kurzum: dem nichtrationalen oder gar irtationalem Kapitalismus, beruhte der moderne rationale Kapitalismus für Weber auf System (vgl. WuG: 643 oder WG: 286) und rein ökonomischen Zweck (vgl. WuG: 709; WG: 299).
}

Vor einer solch klaren Verknüpfung von Handlungs- und Strukturebene im Rahmen einer differenzierungstheoretischen Begründung der Entwicklung der modernen Wirtschaft bleiben die neueren sozio-historischen Ansätze weit zurück.

So geht Braudel in vager Anlehnung an Parsons - dessen statische Theorieanlage er ansonsten ablehnt - davon aus, dass jede ,dichte" Gesellschaft sich in anlage er ances Ökonomischen, des Polimehrere Einheiten unterteilen lässt: in den Bereich des Okonomischen, des Politischen, des Kulturellen und des Hierarchisch-Gesellschaftichen (vgl. Braude 1979b/90: 507; Braudel 1979c/90: 44; 1986: 60). Er widmet aber diesem Differenzierungsprinzip keine große Aufmerksamkeit ${ }^{11}$ oder bietet eine Theorie der Ausdifferenzierung mit systematischen Bezügen zur Handlungsebene an. In ähnlicher Weise verfährt Michael Mann. Mann unterscheidet mit ideologischer, ökonomischer, militärischer und politischer Macht und Organisation vier Quellen der Macht und Organisation, die historisch wirkkräftig in der Formung kollektiver Macht sind, die aber keinesfalls für einzelne gesellschaftliche Subsysteme reserviert werden können. Eine systemische Rückbindung an die Handlungsebene fehlt jedenfalls auch hier. In keinem der neueren sozio-historischen Ansätebene fehlt jedenfails auch hier. In keinem der neueren sozio-historischen der Ausze - und schon gar nicht in jentischen Wirtschaft derzeit so konzise theoretisch gefasst und an die Handlungsebene rückgebunden wie bei Weber.

Auch viele der Ansätze der trendorientierten Kapitalismusliteratur sind nur noch auf der Makroebene verankert, ohne Rückbezug auf die Handlungsebene, aber auch ohne eine ausformulierte systemtheoretische Perspektive. So wird zwar konstatiert, dass die Finanz- und Kommunikationsrevolution es den nationalen Wirtschaften schwer mache, ihre Geschicke selbst zu bestimmen (vgl. z.B. Reich 1993; Narr/Schubert 1994; Ohmae 1995) oder dass die symbiotische, staatlich vermittelte Verbindung von Finanz- und Industriekapitalismus im „orstaatlich vermittelte Verbindung von Finanz- und Industrickapitalismus in "organisierten Kapitäles $\mathrm{Maß}$ der Ausdifferenzierung des Finanzkapitalismus nach kenwesens ein habe (vgl. Lash/Urry 1987: 209), aber Bezugnahmen zu den Träsich gezogen habe ( Kalkülen bleiben aus. Bei gerschichten und iuber den ,Kasino“- bis hin zum „neuen Kapitalismus“ (Sennett 1998a,b; 2005; Strange 1997 etc.) - macht sich das Fehlen einer Kapitalismustheorie von der Reichge 1997 etc.) - macht sich das Fehlen einer Kapitalismustheorie von der Reichweite der Marxschen oder Weberschen ebenso bemerkbar wie die Tatsache, dass
dieser Wandel selten auf der Ebene der Akteure in Organisationen, d.h. auf der Ebene der alltäglichen wirtschaftlichen Deutungen und Orientierungen in den Betrieben untersucht wird. "Braudel selbst benennt dieses Prinzip nie als ,funktionale Differenzierung" und gerät auch häufiger
in terminologische Schwierigkeiten (vgl. nur Braudel 1979c/90: 68, 698). 
Obwohl also die gegenwartsbezogene neuere Kapitalismusliteratur in ihren empirischen Fundiderungen ein differenzierungstheoretisches Verständnis des Kapitalismus nahe llegt, wird dieses nicht nur selten theoriesystematisch ausgefuhrt, sondern hängt ohne simnvollen Handlungsbezug oder einen entsprechenden Theorierahmen in der Luft.

\subsection{Kapitalismusentwicklung als Entwicklung des okzidentalen Rationalismus}

Weber konzentrierte sein Verständnis der Heraufkunft und der gegenwärtigen Bedeutung des modernen rationalen Kapitalismus ébenso einseitig wie Marx dies tat auf die Industrie- und Massenproduktion. Darin macht sich die historische Gebundenheit seiner Annahmen bemerkbar ${ }^{12}$. Andere gegenwärtige Formen des Wirtschaftens gerieten davor eher aus dem Blick. Dabei ist seine historische Beschreibung auf der einen Seite richtig und für seine Zeit zurecht auf das zentrale Merkmal der kapitalistischen Wirtschaft seiner Zeit konzentriert. Auf der anderen Seite erscheint diese historische Verengung aus heutiger Sicht als fragwürdig, als ein Produkt der Kapitalismustheorie jener Zeit, an der die heutige ihre Revisionen orientiert.

Braudels Konzept ist im Gegensatz dazu konsequent in der Sphäre des Warenaustausches, i.e. in der Zirkulationssphäre, angesiedelt. Er verzichtet damit auf ein Verständnis von Kapitalismus, das den „wahren Kapitalismus“ erst mit seinem Übergreifen auf das Produktionssystem am Werke sieht. Erst spät und mit vielen Rückzügen griff nach Braudel der Kapitalismus massiv auf diesen Sektor ein (vgl: Braudel 1979c/90: 696). Den modernen rationalen Kapitalismus auf den Industriekapitalismus und die Fabrikorganisation begrifflich zu konzentrieren (und darauf zu reduzieren), erschien ihm unangemessen. Er trifft sich hier mit der gegenwartsbezogenen Kapitalismusliteratur, bei der mit dem Auseinandertreten von Geld- und Güterströmen sowohl die Bezugnahmen auf die Zirkulationssphäre an Bedeutung gewinnen als auch die Kontingenz der Warenproduktion für den Finanzkapitalismus sichtbar gemacht wird. Sei es das Auseinandernehmen von Produktivkraftentwicklung und Produktionsverhältnissen bei Daniel Bell in den 70er Jahren, das es möglich machte, modernen Kapitalismus und Industrie nicht mehr als eins zu denken (vgl. Bell 1973/85: 11 ff.), seien es in den 80er Jahren die Thesen von Piore/Sabel zum möglichen Ende der Massenproduktion (vgl. Piore/Sabel 1985) und die daran anschließende Postfordismusdiskussion (vgl. für Zusammenfassungen Hirst/Zeitlin 1991; Amin 1994 u.v.a.), jedes Mal werden Trends in den Mittelpunkt gerückt, die einen Kapitalismus als

\footnotetext{
${ }^{12}$ Es war die ,unpersönliche, anethische ökonomische Kapitalherrschaft“ in der modernen großindus-
} triellen Massenproduktion, die Weber vor allem anderen im Blick hatte (WuG: 709, 2l1).
Wirtschaftsformation ohne industrielle Massenproduktion vorstellbar machen. In dem Szenario „desorganisierten Kapitalismus" (vgl. Lash/Urry 1987: $300 \mathrm{ff.}$ wird dieser zudem als losgelöst vom traditionellen Gegensatz von Kapital und Arbeit gedacht. Die vor allem in der Tradition der deutschen Kapitalismustheorie liegenden Fixierungen werden also - oft klammheimlich - uber Bord geworfen, wenn aktuelle Entwicklungen skizziert werden

Dabei fehlt der neueren Kapitalismusliteratur aber eine Unterlegung mit einer Theorie wirtschaftlicher Rationalisierung, vor deren Hintergrund die von ihr konstatierten Trends einschätzbar werden. Auch wenn Webers Konzentration auf die industrielle Massenproduktion heute als fragwürdig erscheint, so stellt seine Theorie des okzidentalen Rationalismus gleichwohl einen wichtigen Baustein für ein modernes wirtschaftssoziologisches Verständnis des Kapitalismus dar und kann den empirischen Befunden der neueren Kapitalismusliteratur eine theoretisch-instruktive Rahmung geben.

Der Prozess okzidentaler Rationalisierung konstituiert sich für Max Weber zuallererst durch die Freisetzung rein formaler Vernunft. Diese Freisetzung heißt nun aber auch, dass sich Handiungsschranken gegenüber direkten Interventionen anderer Teilsysteme gesellschaftlich entfalten lassen. Die Eigenlogiken der jeweiligen Handlungsbereiche können nun auch gegen andere Wertorientierungen legitimerweise verfolgt werden (vgl. Tyrell 1978: 183 f.). Diese Differenzierung in eigenlogische Wertsphären wird bei Weber allerdings an die alltägliche Lebensführung rückgebunden. Weber beobachtet, wie sich in allen Lebenssphären die „Apparatur" der Bürokratie als universelles Mittel der formalen Vernunft ausbreitet. Sie ist für ihn nicht nur eigentümlichster Ausdruck des Prozesses der okzidentalen Rationalisierung, sondern vor diesem Hintergrund auch die Herrschaftsapparatur, derer sich der Handelnde zugleich bedient und zu erwehren hat. Mit ihr verbindet Weber die Gefahr, dass sich die Mittel gegen die Zwecke verselbständigen, dass formale Vernunft in materiale Unvernunft umschlägt. In der Bürokratie liegt für Weber theoriearchitektonisch eine der zentralen Brücken, die zwischen formaler Vernunft und Herrschaft als zwei der wichtigen Elemente im Prozess okzidentaler Rationalisierung vermittelt. Sein differenzierungstheoretisches Verständnis des Kapitalismus wird hier also eingeordnet und fundiert durch eine Theorie okzidentaler Rationalisierung. Dass Weber sein Werk mit der Kapitalismusanalyse beginnt (vgl. für diese Interpretation Schluchter 1979: 15 f.) und erst später in eine allgemeinere sozio-historische Theorie okzidentaler Rationalisierung, ihrer Handlungsorientierungen, Ordnungs- und Strukturprinzipien überführt, sollte also nicht dazu führen; den rationalen Betriebskapitalismus als Explanans zu überschätzen. Der okzidentale Rationalismus, so formuliert es Schluchter pointiert, geht im rationalen Kapitalismus nicht auf. „Dieser ist vielmehr nur eine von mehreren möglichen institutionellen Übersetzungen der Idee 
der Weltbeherrschung" (ebd.: 101). Weber teilt explizit - ich schließe mich hier Schluchters Interpretation an - die bei Marx schwach angedeutete Auffassung vom historischen Zusammenhang zwischen Kapitalismus und Bürokratisierung und seiner revolutionierenden Wirkung, die auf alle Lebensbereiche der Gesellschaft ausstrahlt (vgl. Schluchter 1980: 81). Dieser Zusammenhang ist aber seinerseits wiederum Ergebnis einer Rationalisierung der Lebensfuihrung, welche die okzidentale Rationalisierung viel weitreichender kennzeichnet als bloß unter jenem Aspekt.

Entscheidend fur Weber ist die „Eigenart des Rationalisierungsprozesses", also die. Frage, welche Sphären in welche Richtung rationalisiert werden und damit, in welchen Handlungskontexten welche Rationalitätskriterien handlungsleitend werden können (Lepsius 2000: 34). Weber bietet hier eine theoretische Klammer auch für - in der historischen Erklärung des Kapitalismus anders votierende - Ansätze, die aber im Begreifen des dahinter stehenden Rationalismus regelmäß̣ig zu kurz greifen. So bleibt z.B. Braudels Analyse, die ja ohne - wenn auch implizit bleibende - Bezugnahmen auf den okzidentalen Rationalismus nicht auskommt, in den Rationalisierungsdimensionen unklar, schwankend und könnte hier am meisten von der analytischen Perspektive Webers profitieren. Auch Michael Manns Rede von der „rationalen Rastlosigkeit“ des Christentums, welche jene des Protestantismus nur als späte Sonderform erscheinen lässt, bleibt eine systematische Fassung des okzidentalen Rationalismus schuldig (vgl. Mann 1991: 240). Für die neuere gegenwartsbezogene Kapitalismusliteratur erscheint der hintergründige Bezugspunkt der Weberschen Kapitalismustheorie auf den Prozess okzidentaler Rationalisierung deswegen als so außerordentlich wichtig, weil es den vordergründigen Unterscheidungen von nachindustriellem Kapitalismus, also von Industrie und Dienstleistungen, von Fordismus und Postfordismus, von Kasinokapitalismus und Finanzkapitalismus, aber auch der Analyse des globalen Kapitalismus eine theoretische und analytische Klammer geben könnte, die hinter diese Unterscheidungen zurückfuiht. Dann kann man z.B. hinterfragen, wie sinnvoll eine sektorenspezifische Unterscheidung von Industrie und Dienstleistungen tatsächlich ist, wenn man feststellen kann, dass die Rationalisierungsmodi, Geltungskriterien und Handlungskontexte identisch sind, also die Produktion von Dienstleistungen derselben Rationalität folgt und derselben Logik unterworfen ist wie die Güterproduktion. Dann kann man zwar immer noch Dienstleistungs- und Industrieproduktion unterscheiden, aber nicht mehr zur kulturbedeutsamen Unterschied einer ganzen Gesellschaftsformation heranziehen. $^{13}$

\footnotetext{
${ }^{13}$ Bei allen Unterschieden zeigt sich denn auch die Dienstleistungsarbeit empirisch ähnlichen Rationatisierungskalkülen ausgesetzt wie andere industrielle Segmente (vgl. für eine Zusammenfassung Pohlmann et al. 2003). Eine strukturell angelegte Resistenz gegenüber den finanzwirtschaftlichen
}

Ein differenzierungstheoretisches Verständnis des Kapitalismus heißt bei Weber also nicht, dass die Eigenlogiken der Handlungssphären diese gegeneinander so verselbständigen, dass sie sich in unterschiedlicher Weise dem okzidentalen Rationalismus entziehen. Dies unterscheidet ihn von radikalisierten Fassungen der Differenzierungstheorie, wie sie Luhmann vertritt. Vielmehr ist diéser immer auch in der materiellen Produktion und Reproduktion der alltäglichen Lebenspraxis virulent. ${ }^{14}$

\subsection{Kapitalistische Entwicklung als zukunftsoffener Prozess}

In seiner hiśtorischen Erklärung der Entstehung des Kapitalismus bietet Weber eine vergleichsweise komplexe historische Theorie an, welche die kapitalistische mit den Entwicklungen in den anderen Wertsphären verbindet. Auch sie kann in ihrem Zuschnitt, in ihrer Vorsicht gegenüber "Gesetzmäßigkeiten" ein differenzierungstheoretisches Verständnis des Kapitalismus fundieren helfen.

So ist die viel diskutierte These des Einflusses der "protestantischen Ethik" $m$ E. nur vor dem Hintergrund von Webers Theorie der Ausdifferenzierung der Wirtschaft richtig zu verstehen (vgl. dazu auch Pohlmann 2005). Weber konzentrierte sich auf die rational rekonstruierbare individuelle Eigenart der modernen Kultur (vgl. die Zusammenfassung bei Schluchter 1991: 61-63) und ging von der historischen Einmaligkeit einer solchen Entwicklung aus. ${ }^{15}$ Danit werden z.B. direkte Analogieschlüsse zur Protestantismusthese sinnlos, ${ }^{16}$ und der Ableitung eines generellen Arguments (z.B. dass Kultur wirtschaftliches Verhalten hervorbringe) steht die Einzigartigkeit oder Unwiederholbarkeit des historischen Vor-

Kalkülen oder anderen Rationalisierungsimperativen lässı sich nach neueren Forschungsbefunden nicht erkennen.

${ }_{14}$ Die rationale Gestaltung des äußeren Lebens, so fuhrt Weber diese Position u.a. am Beispiel der Wissenschaft aus, habe wenigstens im Prinzip ihr Werk getan: ,die Unilormierung des äußeren Lebensstils an Hand der 'standardization' der Produktion, ist, unter den heutigen Bedingungen des 'geschäftlichen' Lebens, ihre universelle Wirkung" (GPS: 64).

${ }^{13}$ Dies ist eines der Denkprinzipien einer historischen Interpretation, die Weber als Prämisse seines Werkes ansiebt (vgl. dazu z.B. Schluchter 1979: 23 ff.; Habermas 1981: 220 f.; Mommsen 1982: 189 f.) Die wissenschafliche Ausgangslage, in der Max Weber die Rationalisierungsthematik aufnimmt, ist durch die Kritik an den Evolutionstheorien des 19. Jahrhunderts bestimmt (vgl. Habermas 1981 220). Er richtet sich gegen die Annahme von Entwicklungsgesetzen, gegen dialektische sowie evolutionistische Geschichts- und Gesellschaftstheorien und arbeitet dagegen die Eigenart von Kultur als tionistische Geschichts- und Gesellschaftstheorien und arbeitet dagegen die Eigenart von
eines durch Sinnzusammenhänge konstituierten Gegenstandsbereichs heraus (vgl. ebd.).

${ }^{16}$ Zwes durch Sirnzusammenhänge konstituierten Gegenstandsbereichs heraus (vgl. ebd.).
${ }^{2}$ man Webers Werk als Aufforderung verstehen, nach analogen Entwicklungen in anderen Kulturen zu suchen (vgl. Schluchter 1979: 37), aber dazu müsste man die jeweils individuellen institutionellen Voraussetzungen qualifizierend miteinbeziehen, die den Konfuzianismus dann über seine Verwirklichungsbedingungen zu etwas anderem machen als zu einem funktionalen Äquivalen zum Protestantismus. 
gangs gegenuiber. Mit Webers Vorstellungen zur kapitalistischen Entwicklung wird es deshalb möglich, eine finale Fassung von Kapitalismus ebenso wie Vorstellungen linearer bzw. gerichteter Entwicklung (wie sie im Mainstream der älteren Kapitalismustheorie gerne gepflegt wurden) ganz aufzugeben. Dies ist instruktiv für die neuere Kapitalismusliteratur, die sich bezuiglich einer Vorstellung kapitalistischer Entwiclelung, die auf ein (sich mehr oder weniger zwangsläufig einstellendes) Ende oder einen Übergang spekuliert, ohnehin unsicher geworden ist.

Mit den Spekulationen über das Ende der Massenproduktion, über einen postindustriellen oder postfordistischen Kapitalismus wird in der neueren Kapitalismusliteratur zugleich die Zukunft des Kapitalismus - mit wenigen Ausnahmen (vgl. z.B. Drucker 1993) - offen gehalten wird. Seit man begonnen hat, sich intensiver mit den Wandlungen des Kapitalismus auseinander zusetzen, schein sein Ende ebenso unsicher geworden zu sein wie sein Anfang. Vorstellungen von linearer Entwicklung oder Höherentwicklung jedenfalls haben sich verloren. Die neuere Kapitalismustheorie beschränkt sich darauf, den Wandel wissenschaftlich in den Begriff zu bekommen und nicht mehr darüber hinaus das nahe Ende des Kapitalismus zu deduzieren. Selbst auf marxistischer Seite - bei Wallerstein oder den Vertretern der französischen Regulationstheorie - wird die Zukunft heute eher offen gehalten. Wenn noch in dieser Tradition über das Ende des Kapitalismus spekuliert wird, dann ist es, seltsam genug, der Markt, der dieses Ende bereitet (vgl. Wallerstein 1995: 246 f.), oder man wartet - wie die französische Regulationstheorie - auf die Fortsetzung des Kapitalismus mit anderen Mitteln Die Probleme, die aus diesem Offenhalten resultieren, sind jedoch beträchtlich Eine schnell postulierte Prognose oder Trendprojektion jagt die nächste und die in der historischen Analyse der Entwicklung des Kapitalismus liegenden Wurzeln wurden - vermutlich aus Gründen der eher ungewollten, gezwungenen Verabschiedung der Finalitätskonzeption des Kapitalismus - weitgehend gekàppt.

Eine Bezugnahme auf.Webers Entwicklungstheorie könnte hier helfen, die Prämissen eines differenzierungstheoretisches Verständnisses von kapitalistischer Entwicklung genauer zu fassen. Denn diese besagt in pointierter Weise was auch für neuere Evolutionstheorien heute selbstverständlich geworden ist: dass eine Entwicklungstheorie sich am besten als eine Theorie des geschichtlich einmaligen Aufbaus von Systemen versteht, und formuliert die Annahme, dass Entwicklung durch eine Nutzung von vorübergehenden, nicht bleibenden Bedingungen zustandekommt (vgl. dazu auch Luhmann 1997: 416). Nimmit man dies ernst, liefert z.B. der in der Diskussion um die Bedeutung von Arbeitsethiken oder religiöse Werte gepflegte Rückverweis auf Weber kein Argument mehr und man muss die Entwicklungsannahmen sowohl auf eine Differenz zwischen ge- sellschaftlicher und kapitalistischer Entwicklung als auch die Unwiederholbarkeit von Entwicklung einstellen. Einfache Vorstellungen von nachholender Entwicklung werden damit ebenso gekappt wie kapitalistische Entwicklung dann nur noch auf Basis gesellschaftlicher Differenzienung vorstelibar wird. Die allzu einfache Verbindung zwischen kulturellen Werten und kapitalistischer Entwicklung, die gerade auch Teile der neueren Kapitalismusliteratur kennzeichnet (vgl. z.B. Fukuyama 1995), ist damit auf die Berücksichtigung der gesellschaftsstrukfurellen Voraussetzungen für die Entwicklung des Wirtschaftssystem verwiesen, um die Bedeutung religiöser Werte oder Arbẹitsethiken überhaupt abschätzen zu können.

Webers, Entwicklungsprognosen selbst geben nur soziologische Wahrscheinlichkeiten an, denen unvermeidbar ein Moment der Spekulation innewohnt ${ }^{17}$ (vgl. dazu auch Schluchter 1980: 143). Dabei tendiert für Max Weber die alltägliche Lebenspraxis, so seine seit 1905 immer wieder gezogene Schlussfolgerung, mit deŕ okzidentalen und kapitalistischen Entwicklung zu einer Form zu gerinnen, die Weber bekanntermaßen als "Gehäuse firr die neue Hörigkeit" bezeichnet hat ${ }^{18}$. Dass er zu diesen gerade auch Prozesse der Entdifferenzierung von Wertsphären rechnet, eine übergreifende Standardisierung der Lebensführung, die in der Verschmelzung von Kapitalismus und bürokratischer Herrschaft im Prozess okzidentaler Rationalisierung ihre Zuspitzung erfährt, zeigt an, was für ein differenzierungstheoretisches Verständnis kapitalistischer Entwicklung besonders wichtig ist: Die Analyse ihrer Dynamik muss auf die institutionellen Konfigurationen einer differenzierten Gesellschaft bezogen werden. Jede Kapitalismustheorie, die Entwicklung allein auf wirtschaftliche Trends bezieht und nur darin ihre Dynamik verankert, greift ebenso zu kurz wie jede einseitig auf Politik bezogene. Kapitalistische Entwicklung muss mit Weber auf das konfliktreiche Zusammenwirken institutioneller Ordnungen bezogen werden und daraus ihre Bestimmung ziehen. Das zumindest fordert ein differenzierungstheoretisches Verständnis kapitalistischer Entwicklung. Weber jedenfalls demonstrierte dieses auch in seinen gesellschaftspolitischen Schlussfolgerungen, indem er vor allem

${ }^{17}$ Denn, so Max Weber, über die kausale Bedeutung der Tatsachen der Gegenwart als ,Ursachen ,entscheidet' endgültig erst die Zukunft und der Übergang von rationaler zu spekulativer Kalkulation ist völlig flüssig, da keine auf die Zukunft abgestellte Berechnung von unerwarteten Zufällen objektiv gesichert ist. (WuG: 92)

${ }^{18}$ In seiner Abhandlung über die bürgerliche Demokratie in Russland benennt er die Prozesse, die eine solche Entwicklung vorantrieben genau. Damit das überall - „im amerikanischen ,benevolent feudalism", in den deutschen 'Wohlfahrtseinrichtungen', in der "russischen Fabrikverfassung" bereits fertige Gehäuse der Hörigkeit seines Erachtens bezogen werden kann, bedarf es nur noch einer „Verlangsamung im Tempo des technisch-ökonomischen 'Fortschritts" und des Siegs ", de 'Rente' über den 'Gewinn' in Verbindung mit der Erschöpfung des noch 'freien' Bodens und der noch 'freien' Märkte, um die „Massen 'gefügig' zu machen, es endgültig zu beziehen" (GPS: 63, Hervorh. im Original, vgl. dazu auch ausführlich SWG: 277 f.) 
die Rücknahme gesellschaftlicher Differenzierung durch die universellen Wirkungen des okzidentalen Rationalismus als tendenziell verhängnisvoll ansah und das Individuum - auf dessen Strukturierungskraft er zugleich seine Hoffnung setzte - bedroht, davor zurückzubleiben.

\section{Nach Weber: Zu den Möglichkeiten einer reformulierten Kapitalismustheorie}

Einige zentralen Grundannahmen des Mainstreams der marxorientierten deutschen Kapitalismustheorie des vergangenen Jahrhunderts haben heute sichtlich an Erklärungskraft verloren. Nicht nur die phasen- und krisenförmig gedachte Theorie gerichteter kapitalistischer Entwicklung, nicht nur die anhängigen Konvergenz- oder Dependenzvorstellungen und nicht nur die Entwicklungsform als Klassengesellschaft erwiesen sich zunehmend als konzeptionelle Sackgassen, in denen sich die sozialwissenschaftliche Diskussion buchstäblich festgefahren hat. Auch das Amalgamieren von Wirtschaft und Gesellschaft, das seinen Ausdruck und seinen Auspruch in der Rede von der kapitalistischen Gesellschaft findet, sucht konzeptionell über die Klassiker und ihre Epigonen hinaus nach Anschlussmöglichkeiten. Der Weg aus diesen konzeptionellen Schwierigkeiten wird derzeit eher in pragmatischer, denn in theorieorientierter Weise gesucht. Insbesondere die anglo-amerikanische Kapitalismusliteratur war in den letzten drei Jahrzehnten bestrebt, sowohl in ihren historischen als auch in ihren Gegenwartsanalysen und Trendprojektionen Korrekturen an den Traditionen der Kapitalismustheorie anzubringen. Dabei fehlt ihr jedoch eine Theorie zur Einordnung ihrer Befunde und damit eine Verständigungsbasis, um die Kluft zwischen strukturaler Geschichte und gegenwartsbezogenen Analysen des Kapitalismus zu überbrücken.

Die pragmatische anglo-amerikanische Kapitalismusliteratur lässt zwar die soziologische Klassik der Kapitalismustheorie teilweise als empirisch überhoit erscheinen und trifft sich hier, bezogen auf den Marxschen Kanon der Kapitalismustheorie, mit Luhmanns Interpretation, für den der unbestreitbare Erfolg dieser Theorie sich einer Konjunktur, einer relativen historischen Übereinstimmung von Idee und Realität verdanke, aber diese Konjunktur vergangen sei (vg). Luhmann 1988: 163). Sie macht aber derzeit kein theoriesystematisches Angebot, dass dem empirischen Überholen einiger zentraler Annahmen der deutschen Klassik der Kapitalismustheorie eine ähnlich starke konzeptionelle Fassung gäbe. Hier setzt mein Vorschlag an, vor dem Hintergrund dieser theoriesystematischen Lücke eine Kapitalismustheorie fortzuführen, wie sie Weber und Sombart begonnen haben und dieser also ein differenzierungstheoretisches Verständnis des Kapitalismus zu unterlegen. Im Zuge dieser Umstellung kann versucht werden, die Kapitalismustheorie von ihrer gesellschaftstheoretischen Überfrachtung und überschießenden politischen Konnotationen zu befreien und anschlussfähig für einen modernen wirtschaftssoziologischen Diskurs zu machen, ohne aber die Bezüge zur Gesellschaftstheorie aufzugeben. Dabei war nicht die Überführung einer Theorie in die andere oder beider in eine das Ziel, sondern zu prüfen, inwieweit die Kapitalismustheorie heute vom Kalkül einer Differenzierungstheorie, wie sie Weber angelegt hatte, und ihren Entwicklungsannahmen profitieren kann.

Wir haben dabei zunächst im Anschluss an Sombart gesehen, dass es nicht nur - wie' insbesondere von der gegenwartsbezogenen Kapitalismusliteratur betont - empirisch gerechtfertigt ist, sondern sich auch lohnt, den Kapitalismus nur als eine Wirtschaftsweise unter anderen zu begreifen, die mit diesen koexistiert. Denn erst durch diese Rücknahme wird es möglich, seinen Terraingewinn, seine Kolonialisierungsversuche und Übergriffe einzublenden und auch zu sehen, wo er mit institutionalisierten Widerständigkeiten zu rechnen hat. In einer holistischen oder monistischen Gleichsetzung von Kapitalismus und Gesellschaft wäre diese Perspektive obsolet. Zugleich eröffnet diese Rücknahme die Möglichkeit eines historischen Vergleichs der institutionellen Bedingungen der Entfaltung des Kapitalismus unter der Frage, die auch für die heutige Analyse des modernen, rationalen Kapitalismus von großer Relevanz ist: Unter welchen historischen Bedingungen und institutionellen Konfigurationen lassen sich die Geltungsansprüche des Kapitalismus im Sinne einer "Ökonomisierung der Gesellschaft" verwirklichen und welche Trägerschichten treiben diese mit welchem Kalklil voran? Erst auf Basis eines differenzierungstheoretischen Verständnisses des Kapitalismus kann man diesen als ,umkämpftes gesellschaftliches Terrain“ einblenden und seine Entfaltung unter Bezug 'auf die Handlungs- und Strukturebene erklären. Auch hier erweisen sich die Vorteile eines Rückgriffs auf die Weberschen differenzierungstheoretischen Überlegungen als schlagend ${ }^{19}$. Weder die neuere Socio-Histoire leistet derzeit eine Verknüpfung von. Handlungs- und Strikturtheorie noch die gegenwartsbezogene Kapitalismusliteratur, die spekulativ ganz der Makroebene verhaftet bleibt. Dort, wo der konstatierte Wandel des Kapitalismus seine ,interne" Verankerung hat und wo umgekehrt neue Deutungsmuster und Kalküle als Impulsgeber auf ihn einwirken, bleibt er zumeist unbestimmt und die organisierten Handlungsrationalitäten seiner Trägerschichten ausgeblendet.

${ }^{19}$ Dies auch deshalb, weil ein einfacher Zugriff auf "reine" Theorie gesellschaftlicher und sozialer Differenzierung derzeit nur schwer möglich ist. Sie weist noch immer - wie Tyrell bereits 1978 konstatierte (vgl. Tyrell 1978: 176) - beträchtliche Unschärfen und Ausarbeitungsdefizite auf (vgl. daza heute auch Türk 1995; Schwinn 1995a, 1995b, Tyrell 1998a, 1998b). 
Als Hintergrundthema, in das ein differenzierungstheoretisches Verständnis des Kapitalismus eingebettet ist und das diesem einen genau definierten gesellschaftstheoretischen Bezug gibt, bietet sich Webers Theorie der okzidentalen Rationalisierung an. Sein Verständnis des okzidentalen Rationalismus liefert eine Bestimmung von Analysedimensionen, die mit der Bezugnahme zur Handlungsebene heute helfen können, die neuere Kapitalismusliteratur zu orientieren und ihren Diskurs im ,dreipoligen Raum von Handlungsabläufen, Strukturbildungen und Sinnprojektionen" (Lepsius 2000; 33) des okzidentalen Rationalismus zu verorten. Dabei wird es zugleich wichtig, auf eine Theorie kapitalistischer Entwicklung umzustellen, die nicht nur die Vorsichtsmaßnahmen Webers in der historischen Argumentation berücksichtigt, sondern deren Bezugspunkt auch in einer Differenzierung von Wertsphären sieht. Diese befördern keineswegs konfliktfrei oder gleichgerichtet den modernen Kapitalismus. Seine Entwicklung wird vielmehr von ihrem widersprüchlichem Zusammenspiel in Form von institutionellen Konfigurationen bestimmt. Dies zu berücksichtigen, fällt der neueren Kapitalismusliteratur bislang schwer, verzichtet sie doch weitgehend auf institutionenbezogene Analysen, die deren Zusammen- und Entgegenwirken einblenden. Erst neuere institutionalistische Ansätze wie jener von Hall/Soskice (2001) beginnen langsam, hier ein Problembewusstsein zu schaffen, das einem differenzierungstheoretischen Verständnis kapitalistischer Entwicklung entspricht. Aber der Weg zu einer klar gefassten theoretischen Perspektive ist hier noch weit und die Kapitalismustheorie täte gut daran, diesen Weg - zumindest ein Stück weit auf den abgesicherten Pfaden des Weberschen Forschungsprogramms zu gehen.

\section{Literatur}

Albert, Gert (2005): Moderater methodologischer Holismus. Eine weberianische Interpretation des Makro-Mikro-Makro-Modells. In: Kölner Zeitschrift für Soziologie und Sozialpsychologie 57. (3). 2005. 387-413

Amin, Ash (Hrsg.)(1994): Post-Fordism. Oxford (UK). Cambridge (USA): Blackwell

Bell, Daniel (1973/85): Die nachindustrielle Gesellschaft. Frankfurt/M., New York: Campus

Braudel, Fernand (1979b/90): Sozialgeschichte des 15.-18. Jahrhunderts. Der Handel München: Kindler

Braudel, Fernand (1979c/90): Sozialgeschichte des 15.-18. Jahrhunderts. Aufbruch zur Weltwirtschaft. München: Kindler

Braudel, Fernand (1986): Die Dynamik des Kapitalismus. Stuttgart: Klett-Cotta

Crow, Graham (1997): Comparative Sociology and Social Theory. Beyond the Three Worlds. Houndmills et al: Macmillan

Drucker, Peter (1993): The Postcapitalist Society. New York: Harper Business
Fukuyama, Francis (1995): Konfuzius und Marktwirtschaft. Der Konflikt der Kulturen München: Kindler

Habermas, Jürgen (1981): Theorie des kommunikativen Handelns. Bd. 1: Handlungsrationalität und gesellschaftliche Rationalisierung. Frankfurt/M.: Suhrkamp

Habermas, Jürgen (1985): Der philosophische Diskurs der Moderne. Zwölf Vorlesungen. Frankfurt/M.: Suhrkamp

Hall, Peter A., David Soskice (Hrsg.) (2001): Varieties of Capitalism. The Institutional Foundations of Comparative Advantage. Oxford: Oxford University Press

Hilferding, Rudolf (1910/73): Das Finanzkapital: Frankfurt/M.: Europäische Verlagsanstait

Hirst, Paul, Zeitlin, Jonathan (1991): Flexible Specialization versus Post-Fordism. Theory, Evidence, and Policy Implications. In: Economy and Society. Vol. 20. No.1. 1991. I 56

Lash, Scott, John Urry (1987): The End of Organized Capitalism. Cambridge: Polity

Lenin, Wladimir I. (1917/83): Der Imperialismus als höchstes Stadium des Kapitalismus. 18. Aufl., Berlin-Ost: Dietz Verlag

Lepsius, Rainer M. (2000): Eigenart und Potenzial des Weberparadigmas. In: Albert, Gert et al. (Hrsg.): Das Weber-Paradigma. Studien zur Weiterentwicklung von Max Webers Forschungsprogramm. Tübingen: Mohr Siebeck. 32-41

Luhmann, Niklas (1985): Zum Begriff der sozialen Klasse. In: Luhmann, Niklas (Hrsg.) (1985): Soziale Differenzierung. Zur Geschichte einer Idee. Opladen: Westdeutscher Verlag. 119-162

Luhmann, Niklas (1988): Die Wirtschaft der Gesellschaft. Frankfurt/M.: Suhrkamp

Luhmann, Niklas (1994): Soziologische Aufklärung 4. Beiträge zur funktionalen Differenzienung der Gesellschaft. 2. Auflage. Opladen: Westdeutscher Verlag

Luhmann, Niklas (1997): Die Gesellschaft der Gesellschaft. 2..Bde. Frankfurt/M.: Suhrkamp

Luxemburg, Rosa (1913): Die Akkumulation des Kapitals. Berlin: Vorwärts

Mann, Michael (1990): Geschichte der Macht. Bd. 1. Von den Anfängen bis zur griechischen Antike. Frankfurt/M., New York: Campus

Mann, Michael (1991): Geschichte der Macht. Bd.2. Vom Römischen Reich bis zum Vorabend der Industrialisierung. Frankfurt/M., New York: Campus

Marx, Karl(1873), Das Kapital. Bd.1. Nachwort zur zweiten Auflage. London

Marx, Karl (1890/1976): Das Kapital. Kritik der politischen Ökonomie, Hrsg. v. Friedrich Engels, Erster Band. 4. Aufl. MEW 23. Berlin: Dietz Verlag [zit. KI]

Marx, Karl (1893/1977): Das Kapital. Kritik der politischen Ökonomie. Hrsg. v. Friedrich Engels, Zweiter Band. MEW 24. Berlin: Dietz Verlag [zit. KII]

Marx, Karl (1894/1972): Das Kapital. Kritik der politischen Ökonornie. Hrsg. v. Friedrich Engels, Dritter Band, MEW 25. Berlin: Dietz Verlag [zit. KIII]

Marx, Karl (1953/74): Grundrisse der Kritik der politischen Ökonomie (Rohentwurf 1857/58). Berlin: Dietz Verlag [zit. GpÖ]

Marx, Karl (1962): Manifest der kommunistischen Partei. MEW 4. Berlin: Dietz Verlag [zit. MkP]

Marx, Karl, Friedrich Engels (1962): Die deutsche Ideologie. MEW 3. Berlin: Dietz Verlag [zit. DI] 
Mommsen, Wolfgang (1982): Max Weber. Gesellschaft, Politik und Geschichte. Frankfurt/M.: Suhrkamp

Narr, Wolf-Dieter, Alexander Schubert (1994): Weltökonomie. Die Misere der Politik. Frankfurt/M.: Suhrkamp

Ohmae, Kenichi (1995): The End of the Nation State. The Rise of Regional Economies. New York et al.: Free Press

Parsons, Talcott (1928): Capitalism in Recent German Literature. Sombart and Weber (I). In: The Journal of Political Economy. Vol. 36. No. 6. 1928. 641-661

Piore, Michael J., Sabel, Charles F. (1985): Das Ende der Massenproduktion. Studie über die Requalifizierung der Arbeit und die Rückkehr der Ökonomie in die Gesellschaft Berlin: Wagenbach

Pohimann, Markus (1996): 'Nach Weber': Sozialwissenschaftliche Interpretationen der Zukunft des Kapitalismus. Berliner Debatte Initial, Vol. 7, No. 6. 1996. 54-66

Pohlmann, Markus (2005): Die neue Kulturtheorie und der Streit um Werte. Soziologische Revue. 28. No.1. 2005. 3-14

Pohlmanu, Markus et al. (Hrsg.) (2003): Dienstleistungsarbeit - Auf dem Boden der Tatsachen. Berlin: edition sigma

Reich, Robert (1993): Die neue Weltwirtschaft. Das Ende der nationalen Ökonomie. Frankfurt/M.: Fischer

Runciman, W. G. (1989): A Treatise on Social Theory. Vol. II: Substantive Social Theory. Cambridge: Cambridge University Press

Schluchter, Wolfgang (1979): Die Entwicklung des okzidentalen Rationalismus. Tübingen: Mohr

Schluchter, Wolfgang (1980): Rationalismus der Weltbeherrschung. Studien zu Max Weber. Frankfurt/M.: Suhrkamp

Schluchter, Wolfgang (1991): Religion und Lebensfuhrung. Bd. 1. Studien zu Max Webers Kultur- und Werttheorie. Frankfurt/M.: Suhrkamp

Schluchter, Wolfgang (2000): Handlungs- und Strukturtheorie nach Max Weber. In Berliner Journal fúr Soziologie. Vol. 10. No. 1. 2000. 125-136

Schumpeter, Joseph A. (1942/93): Kapitalismus, Sozialismus und Demokratie. 7. erw. Auflage, Tübingen und Basel: Francke (UTB)

Schwinn Thomas (1995a): Funktionale Differenzierung - wohin? Eine aktualisierte Bestandsau fnahme. Berliner Journal fuir Soziologie. No. 1. 1995. 25-39

Schwinn, Thomas (1995b): Funktion und Gesellschaft. Konstante, Probleme trotz Paradigmenwechsel in der Systemtheorie Niklas Luhmanns. In: Zeitschrift für Soziologie. Vol. 24, No. 3. 1995. 196-214

Sennett, Richard (1998a): Der flexible Mensch. Die Kultur des neuen Kapitalismus. Berlin: Berlin Verlag

Sennett, Richard (1998b): Der neue Kapitalismus. In: Berliner Journal fur Soziologie. Vol. 8. No. 3. 1998. 305-316

Sennett, Richard (2005): Die Kultur des neuen Kapitalismus. Berlin: Berlin Verlag

Skocpol, Theda (1979): States and Social Revolutions. A Comparative Analysis of France, Russia, and China. Cambridge: Cambridge University Press

Sombart, Werner (1927/87): Der moderne Kapitalismus. Bd. III, 1. München: DTV [zit. MK III, 1]
Sombart, Werner (1927/87): Der modeme Kapitalismus. Bd. III, 2. München: DTV [zit. MK III,2]

Strange, Susan (1997): Casino Capitalism. Manchester. New York: Manchester University Press

Streeck, Wolfgang (1992): Social Institutions and Economic Performance. Studies of Industrial relations in Advanced Capitalist Economies. London et al.: Sage

Türk, Klaus (1995): Die Organisation der Welt..Opladen: Westdeutscher Verlag

Tyrell, Hartmann (1978): Anfragen an die Theorie der gesellschaftlichen Differenzierung. In: Zeitschrift für Soziologie. Vol. 7, No. 2. 1978. 175-1.93

Tyrell, Hartmann (1998a): Werksgeschichtliches zum Kapitalismus. Bielefeld: Ms.

Tyrell, Hartmann (1998b): Zur Diversität der Differenzierungstheorie. Soziologiehistorische Anomerkungen. Bielefeld: Ms.

Vester, Heinz-Günter (1995): Geschichte und Gesellschaft. Ansätze historischkomparativer Soziologie. Berlin, München: Quintessenz

Wallerstein, Immanuel (1995): Die Sozialwissenschaft 'kaputtdenken'. Die Grenzen der Pardigmen des 19. Jahrhunderts. Weinheim: Beltz Athenäum

Weber, Max (1910/82): Die protestantische Ethik I. Hrsg. v. Johannes Winckelmann. 4. erw. Aufl. Gütersloh: Mohn [zit. PI]

Weber, Max (1920/1978): Die Protestantische Ethik I. Tübingen: Mohr [zit. PI]

Weber, Max (1920/1988): Gesammelte Aufsätze zur Religionssoziologie I. 9. unv. Auft. Tübingen: Mohr (zit. RSI)

Weber, Max (1922/855): Wirtschaft und Gesellschaft. Grundriß der verstehenden Soziologie. 5. rev. Aufl, besorgt von J. Winckelmann. Tübingen: Mohr (zit. WuG)

Weber, Max (1923/915): Wirtschaftsgeschichte. Abriss der universalen Sozial- und Wirtschaftsgeschichte. 5. unv. Auflage. Aus den nachgelassenen Vorlesungen. Hrsg. v. S. Hellmann, M. Palyi. Berlin: Duncker-Humblot (zit. WG)

Weber, Max (1924/882): Gesammelte Aufsätze zur Sozial- und Wirtschaftsgeschichte. 2. Aufl. Hrsg. v. Marianne Weber. Tübingen: Mohr (zit. SWG)

Weber, Max (1924/88): Gesammelte Politische Schriften. 5. Aufl. Hrsg. v. Johannes Winckelmann. Tübingen: Mohr (zit. GPS) 